\title{
Evidências de validade baseadas na estrutura interna para escala de atitude empreendedora (ATEBr)
}

\author{
Pedro Afonso Cortez ${ }^{1}$ \\ Universidade São Francisco, Campinas, SP, Brasil \\ Heila Magali da Silva Veiga \\ Universidade Federal de Uberlândia, Uberlândia, MG, Brasil \\ Jonilto Costa Sousa \\ Universidade de Brasília, Brasília, DF, Brasil
}

\section{Resumo}

O empreendedorismo é um fenômeno multideterminado, sendo relevante a compreensão dos aspectos pessoais associados ao tema. O presente estudo objetivou analisar evidências de validade baseada na estrutura interna para uma medida adaptada de atitude empreendedora numa amostra de 272 estudantes universitários. Para análise da estrutura interna da escala foi realizada análise fatorial exploratória. Foi encontrada uma solução fatorial de cinco fatores para o contexto brasileiro (liderança, realização, risco, controle e criatividade). A confiabilidade dos fatores da medida foi estimada por meio dos coeficientes alfa e ômega para dados ordinais variando entre 0,56 e 0,74 . Recomenda-se o uso da escala em estudos ulteriores, a fim de apurar evidências de validade em outros contextos e investigar variáveis antecedentes e consequentes de atitude empreendedora que otimizem as condições de avaliação do empreendedorismo nas práticas de orientação profissional.

Palavras-chave: medidas de atitude; medidas; empreendedorismo; profissões; trabalho

Abstract: Validity evidences through internal structure for entrepreneurial attitude scale (ATEBr)

Entrepreneurship is a multidetermined phenomenon, being relevant the understanding of the personal aspects associated with it. The present study aimed to analyze evidence of validity based on the internal structure for an adapted measure of entrepreneurial attitude in a sample of 272 university students. For the analysis of the internal structure of the scale, an exploratory factorial analysis was performed. It was found a factorial solution of five factors for the Brazilian context (leadership, achievement, risk, control and creativity). The reliability of the measurement factors was estimated by means of the alpha and omega coefficient for ordinal data varying between 0.56 and 0.74 . It is recommended to use the scale in further studies in order to ascertain evidence of validity in other contexts and to investigate relevant variables and consequent of an entrepreneurial attitude that optimize the conditions for evaluating entrepreneurship in professional orientation practices.

Keywords: attitude measures; measures; entrepreneurship; professions; job

Resumen: Evidencia de validez a través de la estructura interna para escala de actitud emprendedora (ATEBr) El emprendedorismo es un fenómeno multideterminado y resulta relevante la comprensión de las características personales implicadas. El presente estudio tuvo como objetivo encontrar evidencias de validez de estructura interna en la escala de medición de actitud emprendedora en una muestra de 272 estudiantes universitarios brasileños. Para analizar la estructura interna de la escala, se realizó un análisis factorial exploratorio. Se encontró una solución compuesta por cinco factores para el contexto brasileño (liderazgo, necesidad de logro, riesgo, control y creatividad). Se estimó la confiabilidad usando coeficientes alfa y omega ajustados a datos de nivel ordinal, varando entre 0.56 y 0.74 . Se recomienda el uso de la escala en estudios posteriores para establecer evidencias de validez en otros contextos e investigar variables antecedentes y consecuentes de actitud emprendedora que optimicen las condiciones de evaluación del emprendedorismo en las prácticas de orientación profesional.

Palabras clave: medidas de actitud; medidas; emprendedorismo; profesiones; trabajo

1 Endereço para correspondência: Universidade São Francisco - Programa de Pós-graduação em Psicologia - Rua Waldemar César da Silveira, 105, Swift, Campinas, SP, CEP: 13045-510.

E-mail: cor.afonso@gmail.com 


\section{Introdução}

O empreendedorismo abrange diferentes áreas de estudo. Dessa forma, compreender as características pessoais que impactam na proposição do negócio pelos indivíduos se propõe como relevante (Inácio, Machado, Gimenez, \& Morini, 2014). Isso acontece, pois, em países de industrialização tardia, como o Brasil, a promoção do empreendedorismo é desejável por impactar nas oportunidades ocupacionais disponíveis para a população geral (Costa-Nogami \& Machado, 2014; Souza, Souza, Pasin, \& Zambalde, 2016).

As contribuições da psicologia para a compreensão do empreendedorismo enfatizam as características pessoais que tornam alguns indivíduos mais ou menos predispostos ao exercício da atividade empreendedora (Frese \& Gielnik, 2014). Entre os diferentes construtos para a compreensão desses aspectos nota-se relevância na atitude empreendedora (Robinson, Stimpson, Huefner, \& Hunt, 1991; Athayde, 2009). Esse construto avalia as atitudes do sujeito em relação a expressar suas características pessoais em contextos associados ao empreendedorismo, sendo estabelecido a partir dessa definição tanto na literatura internacional quanto na nacional (Robinson et al., 1991; Goulart \& Kruger, 2016).

No contexto estudantil, a atitude empreendedora tem se mostrado importante por possibilitar a avaliação da eficácia de práticas voltadas a fomentar a opção ocupacional pelo empreendedorismo (Ismail, 2015). Estudantes com maior nível de atitude empreendedora tendem a intencionar empreender com maior magnitude quando comparados àqueles com baixo nível do construto (Johnson \& Mathew, 2017; Manning \& Parrott, 2017). Numa perspectiva expandida, países em que os sujeitos exibem maiores níveis de atitude empreendedora tendem a apresentar maior nível de atividade empreendedora, o que demonstra a centralidade desse construto para predição do empreendedorismo como alternativa ocupacional (Draghici, Albulescu, \& Tamasila, 2014).

Tal constatação motivou o presente estudo, cujo objetivo foi analisar as evidências de validade empírica baseadas na estrutura interna para uma escala de medida de atitude empreendedora adaptada ao contexto brasileiro (ATEBr - Attitude Towards Entrepreneurship - Brazil). No tópico seguinte são apresentadas considerações acerca das propostas de avaliação do fenômeno adaptada para o contexto brasileiro e, na sequência, os aspectos teóricos relativos à atitude empreendedora que subsidiaram o modelo empregado na investigação.

\section{Medidas de Interesses Profissionais, Empregabilidade e Empreendedorismo no Brasil}

Tratar sobre a avaliação do empreendedorismo envolve, a priori, compreender a distinção entre medidas do fenômeno e aquelas voltadas para interesses profissionais e Empregabilidade. Os interesses profissionais apresentam-se como um padrão motivacional que impele o indivíduo em busca de determinados contextos profissionais. Esses interesses tendem a se mostrar razoavelmente estáveis possibilitando predizer a forma como a pessoa responderá aos estímulos ocupacionais (Rounds $\& \mathrm{Su}, 2014)$. Nesse sentido, essas características tendem a ser apreendidas como tipologias de traços que se associam à opção individual do sujeito sobre o próprio futuro profissional, abrangendo diferentes medidas para avaliação de interesses de forma geral na literatura brasileira (Ambiel, 2016).

Também é possível elencar, entre propostas de avaliação sobre o tema, os instrumentos de Empregabilidade (Cesário \& Feijão, 2014). Essas medidas buscam apreender competências, habilidades e variáveis psicológicas que os indivíduos usam para conquistar e manter os empregos. Alguns instrumentos como as medidas de Autopercepção de Empregabilidade (Peixoto, Janissek, \& Aguiar, 2015) e Escala de Empregabilidade (Campos, 2010) se propõem como alternativas robustas para avaliar Empregabilidade. Nessas escalas a ênfase da avaliação está em compreender de que forma o indivíduo busca por um contexto profissional em detrimento de outro e, também, quais recursos aplica para maximizar a inserção profissional em uma atividade ocupacional em detrimento de outra.

No entanto, tal como nas medidas de interesses, os instrumentos de Empregabilidade se apresentam limitados para mensurações em contextos profissionais específicos, pois tendem a não priorizar a avaliação de fatores ocupacionais predominantes em alguns contextos. Por meio dos aspectos supracitados é possível inferir que as medidas de interesses profissionais e Empregabilidade se apresentam robustas para um rastreamento de interesses numa perspectiva geral. Nesse ponto, encontra-se a diferenciação entre essas medidas e aquelas propostas para avaliar o empreendedorismo. Por se tratarem de medidas dirigidas a um contexto profissional específico, as medidas de empreendedorismo não conseguem diferenciar contextos gerais, mas possibilitam aos orientadores investigar de que forma o indivíduo responderá aos estímulos profissionais em contextos mais restritos (Cortez \& Veiga, no prelo). No caso do presente estudo, enfatiza-se, como contexto específico, a opção ocupacional como empreendedor por meio da proposição do próprio negócio. Nesse sentido, é fundamental a proposição de estudos voltados à mensuração de alternativas ocupacionais em contextos específicos, tal como àqueles relacionados ao empreendedorismo, para possibilitar avanços às práticas de orientação profissional em avaliações direcionadas a cenários ocupacionais mais restritivos (Ambiel, 2017).

Avaliando-se as medidas específicas de empreendedorismo como interesse é possível identificar duas 
tendências, as quais se baseiam nos modelos de traços e atitudes. As avaliações relacionadas aos modelos de traços buscam apreender competências e aspectos psicológicos relativamente estáveis que permitam ao indivíduo empreender com relativo sucesso (Schmidt \& Bohnenberger, 2009). Em contrapartida, os modelos pautados em atitudes reduzem o peso atribuído à avaliação das características pessoais e priorizam o quanto os indivíduos se predispõem a se aproximar de contextos empreendedores ou expressar os atributos psicológicos que possuiao propor o próprio negócio (Robinson, Shaver, \& Wrightsman, 2013). Assim, enquanto as medidas de traços avaliam características pessoais como competências que potencializam ou habilitam o sujeito a empreender, os instrumentos atitudinais voltam-se para a avaliação da predisposição que o sujeito possui para expressá-las em determinado contexto, independentemente do nível de competência que o sujeito possui para empreender (Cortez \& Veiga, no prelo).

Algumas medidas de empreendedorismo sustentadas pelas abordagens de traços podem ser exemplificadas pelas propostas de Souza et al. (2017) e Schmidt e Bohnemberger (2009). Nessas medidas a avaliação do empreendedorismo é enfatizada por meio da caracterização do potencial empreendedor (Souza et al., 2017) e perfil empreendedor (Schmidt \& Bohnenberger, 2009), visando abranger o quanto os indivíduos possuem de traços ou competências que os habilitem empreender com sucesso ou de forma satisfatória.

As propostas ancoradas em atitudes podem ser exemplificas por proposições como Oliveira, Vieira, Laguía, Moriano e Salazar-Soares (2016), Lopez e Souza (2005), Souza, Lopez, Bornia e Alves (2013). Na proposta de Oliveira et al. (2016) a atitude empreendedora é apreendida por meio de um fator geral, o qual se aproxima da proposta de interesses. Isso acontece pois a ênfase do instrumento desenvolvido pelos autores tem como propósito a predição da intenção empreendedora pelo modelo de atitudes de comportamento planejado (TCP), o qual preconiza a atitude como unidimensional. Nessa perspectiva, essa alternativa de mensuração se diferencia da IMAE proposta por Lopez e Souza (2005) pois, apesar de ambas se basearem na concepção de atitudes, a IMAE sustenta-se em uma proposição teórica distinta nomeada modelo tripartite de atitudes.

No modelo tripartite as atitudes podem ser concebidas de forma multidimensional abrangendo afetos, cognições e volições comportamentais relacionadas ao contexto de expressão atitudinal (Chaiken \& Stangor, 1987). Assim, o instrumento desenvolvido por Lopez e Souza (2005) foi hipotetizado por meio de quatro dimensões relativas à atitude empreendedora (realização, planejamento, poder e inovação) para o contexto brasileiro. No entanto, ao realizar a análise fatorial, as evidências empíricas não corroboraram com a fatorabilidade inicial proposta para o modelo, o que possibilita questionar a adequação do instrumento para avaliar a atitude empreendedora por meio do modelo tripartite numa perspectiva multidimensional.

Em estudo posterior, Souza et al. (2013) avaliaram propriedades psicométricas para o instrumento IMAE por meio da Teoria de Resposta ao Item, mas trataram a medida como um construto unifatorial com itens que tinham conteúdos oriundos das quatro dimensões hipotetizadas pelas autoras - realização, planejamento, poder e inovação - deixando uma lacuna nos estudos nacionais sobre a possibilidade de avaliar a atitude empreendedora numa concepção multidimensional. Essa lacuna resultou na análise da literatura internacional em busca de propostas de mensuração da atitude empreendedora sustentadas no modelo tripartite de atitudes que apresentassem replicação de uma estrutura multifatorial. Tal dinâmica resultou na opção de adaptar a ATE (Attitude Towards Entrepreneurship) ao contexto brasileiro, sendo explorados no tópico seguinte os fundamentos dessa proposta de avaliação até a proposição da ATE (Athayde, 2009).

\section{Contribuições Internacionais para Mensuração da Atitude Empreendedora (ATE)}

A atitude empreendedora refere-se à contribuição proposta inicialmente na literatura por Robinson et al. (1991). Esse construto busca avaliar o posicionamento do sujeito frente à expressão das próprias características pessoais em contextos associados empreendedorismo. $\mathrm{Na}$ concepção de Robinson et al. (1991), a atitude empreendedora foi operacionalizada pelas dimensões necessidade de realização, autoestima, autocontrole, inovação e realização. Para isso, Robinson et al. (1991) utilizaram o modelo teórico tripartite de atitudes partindo da noção de que compreender as características pessoais dos empreendedores requeria um modelo atitudinal multidimensional em detrimento aos modelos empregados até então, os quais não se mostravam robustos para a mensuração do fenômeno (Chaiken \& Stangor, 1987; Epstein, 1984).

Em linhas gerais, o modelo tripartite de atitudes avalia o posicionamento do indivíduo em relação aos objetos sociais, abrangendo afetos, cognições e volição comportamental, os quais podem abranger variações positivas, negativas ou neutras (Lindzey, Gilbert, \& Fiske, 1998). Nessa concepção, as atitudes positivas facilitariam a intenção comportamental relacionada ao objeto em análise, ao passo que atitudes negativas diminuiriam a probabilidade de ação relacionada ao fenômeno, enquanto as neutras não exerceriam efeito significativo no comportamento (Ajzen \& Fishbein, 2005).

Tais concepções fomentaram o modelo de mensuração desenvolvido por Robinson et al. (1991) para avaliar a atitude empreendedora. A escala desenvolvida pelos autores, nomeada EntrepreneurialAttitudeOrientation (EAO), contemplou quatro dimensões, que são: realização, 
inovação, autocontrole e autoestima do indivíduo no ambiente de proposição do próprio negócio. Na proposta dos autores, a realização era a busca constante por êxito. A inovação abrangia conteúdos relacionados à inventividade. O autocontrole dimensionava o quanto o indivíduo percebia controlar as próprias manifestações e a autoestima o quanto acreditava ser valorizado por empreender. A consistência interna para os fatores variou entre $0,84 \mathrm{e}$ 0,73 . Contudo, na proposição do estudo de validação da medida, Robinson et al. (1991) não testaram a fatorabilidade das dimensões teorizadas, o que se apresenta como uma lacuna no procedimento empregado pelos autores, a despeito da razoável precisão encontrada para os fatores da medida (Krueger, Reilly, \& Carsrud, 2000; Rauch \& Frese, 2020).

Considerando-se as elaborações mais recentes sobre a atitude empreendedora, identificou-se na contribuição de Athayde (2009) uma adaptação da proposta de Robinson et al. (1991) para o contexto universitário. A autora partiu da noção de que a medida desenvolvida por Robinson et al. (1991) apresentava limitação para aplicação no meio estudantil, uma vez que não versava sobre domínios conhecidos pelos estudantes ao se dirigir contextualmente para negócios. Assim, a autora revisou a literatura em busca de evidências da literatura internacional que relacionavam a atitude empreendedora ao contexto estudantil. Dessa revisão, Athayde (2009) formulou a proposição do ATE em seis dimensões, a partir de estudos empíricos que tratavam sobre o empreendedorismo entre estudantes, a saber: intuição (Gibb, 1987), criatividade (Timmons \& Spinelli, 2004), risco (Carland, Carland, Carland, \& Pearce, 1995), controle pessoal (Krueger \& Brazeal, 1994), realização (Louw, Van Eeden, Bosch, \& Venter, 2003) e liderança (Vecchio, 2003).

No modelo hipotetizado por Athayde (2009), a dimensão intuição refere-se às atitudes voltadas para a preferência pela informalidade em vez da formalidade e à capacidade de lidar com situações incertas para resolver problemas (Gibb, 1987). A criatividade representa atitudes do indivíduo em relação ao quanto ele se considera criativo ou prosaico no ambiente acadêmico (Timmons \& Spinelli, 2004). O risco designa atitudes do indivíduo para assumir riscos em situações com potencial retorno financeiro elevado (Carland et al., 1995). O controle pessoal refere-se à atribuição de causalidade do sujeito e ao quanto ele se percebe como responsável por determinar o próprio futuro profissional (Krueger \& Brazeal, 1994). A realização trata sobre atitudes relacionadas ao orgulho do indivíduo frente à qualidade do trabalho e ao esforço empreendido (Louw et al., 2003). Por sua vez, a atitude relacionada à liderança versa a respeito da percepção do indivíduo quanto às próprias capacidades de influência e direção dos demais (Vecchio, 2003).

Em relação ao estudo de proposição do instrumento e investigação das evidências de validade empírica, Athayde (2009) utilizou análise fatorial exploratória e identificou a estrutura interna da medida em quatro fatores (liderança, criatividade, realização e controle pessoal), a despeito das seis dimensões hipotetizadas, numa amostra de estudantes universitários do Reino Unido. O alfa para os fatores do instrumento variou entre 0,71 e 0,80 demonstrando valores satisfatórios de fidedignidade para a medida, a qual foi empregada no presente estudo por meio da adaptação da medida realizada por Cortez (2017). No tópico seguinte são esclarecidos aspectos relativos ao método empregado na investigação.

\section{Método}

\section{Participantes}

A amostra caracterizou-se como não-probabilística por conveniência. Participaram do estudo 272 estudantes de instituição pública de ensino superior brasileira. Os cursos abrangidos no presente estudo foram administração $(n=34)$; ciências contábeis $(n=26)$; direito $(n=25)$; educação física $(\mathrm{n}=21)$; enfermagem $(\mathrm{n}=30)$; engenharia biomédica $(\mathrm{n}=23)$; medicina $(\mathrm{n}=28)$; nutrição $(\mathrm{n}=26)$; pedagogia $(n=33)$ e psicologia $(n=26)$. Os estudantes cursavam em média o $5^{\circ}$ período $(\mathrm{DP}=2,33)$, sendo predominante a modalidade bacharelado $(58,1 \%)$ e o turno integral $(69,7 \%)$. Em relação às expectativas profissionais dos estudantes, $54,5 \%$ declararam que pretendem criar o próprio negócio e 78,3\% assinalaram que almejam trabalhar como colaboradores em alguma empresa ou governo.

\section{Instrumentos}

O instrumento empregado no estudo foi a versão adaptada ao contexto brasileiro (ATEBr - Attitude Towards Entrepreneurship - Brazil) da medida britânica de atitude empreendedora ATE (Attitude Towards Entrepreneurship) proposta por Athayde (2009). Essa escala foi desenvolvida no estudo original por meio de seis dimensões teóricas (riscos, criatividade, controle pessoal, liderança, intuição e realização) com 36 itens que compõem a atitude empreendedora como construto geral, tendo o conteúdo adaptado para o contexto brasileiro por Cortez (2017). As instruções para preenchimento solicitavam que os estudantes assinalassem em relação a cada um dos itens o quanto concordavam ou discordavam das asserções. A escala de resposta adotadas para os estudantes manifestarem o nível de concordância ou discordância foi do tipo Likert de cinco pontos ( 1 = Discordo plenamente; $5=$ Concordo plenamente).

\section{Procedimentos}

A investigação foi aprovada pelo Comitê de Ética em Pesquisa (CEP) da Universidade Federal de Uberlândia (UFU) sob o número CAAE 56875916.0.0000.5152 
do Sistema Nacional de Ética em Pesquisa (SISNEP). A coleta de dados foi realizada mediante apresentação do Termo de Consentimento Livre e Esclarecido, tal como indicado pela Resolução 510/2016 do Conselho Nacional de Saúde (CNS, 2016). Os instrumentos foram aplicados presencialmente de forma coletiva em sala de aula no intervalo das atividades universitárias. Em média, o tempo de aplicação levou cerca de 20 minutos.

\section{Análise de dados}

A preparação do banco de dados e verificação dos requisitos para execução das análises seguiu as definições estabelecidas por Pasquali (2015) e foi implementada por meio do SPSS (Statistical Package for Social Sciences). O teste Kolmogorov-Smirnov foi utilizado para avaliar a normalidade dos dados. Os índices de KMO e teste de esfericidade de Bartlett foram empregados para analisar a adequação da matriz de dados para análise fatorial. A análise fatorial foi empregada por meio de ESEM (Exploratory Structural Equation Modeling), sendo realizada por meio do softwareMPlus.

Salienta-se que foram seguidas as recomendações definidas por Damásio (2012), o que inclui o uso de estimador para dados ordinais (WLSMV) e correlações policóricas pela natureza das informações aferidas com escalas Likert. O critério de retenção fatorial adotado foi a análise paralela, sendo implementado por meio do pacote 'psych' para R com o uso da função "fa.parallel" para correlações policóricas. Por fim, a consistência interna foi avaliada por meio do alfa de Cronbach e índice Omega.
Esses dois índices foram gerados e ajustados para dados ordinais, com a aplicação do pacote 'user friendly science' para R e a função 'scale Reliability'.

\section{Resultados}

A inspeção inicial dos dados demonstrou pequenos erros de digitação por meio das estatísticas descritivas e apurou violações da normalidade com o teste estatístico Kolmogorov-Smirnov $(p<0,05)$. Nessas condições, foram substituídas pela média as ocorrências omissas e excluídas aquelas com assimetria ou curtose acima de $|2,00|$ de forma a aproximar os parâmetros para razoavelmente próximos à distribuição normal.

Em seguida, inspecionaram-se os índices $\mathrm{KMO}$ $(0,763)$ e o teste de esfericidade Bartlett $(p<0,001)$, os quais demonstraram a adequação dos dados para a utilização da análise fatorial, apesar dos pequenos desvios de normalidade. Em relação à retenção fatorial, a análise paralela indicou evidências favoráveis para a exploração da estrutura adaptada da escala em cinco fatores. Notaram-se autovalores empíricos superiores aos simulados no quinto fator (empírico $=0,96$; simulado $=0,83$ ).

A etapa seguinte foi aplicar a análise fatorial exploratória. O modelo empregado foi do tipo Exploratory Structural Equation Modeling (ESEM). Foram definidos como padrão a exploração da estrutura fatorial em cinco fatores a priori devido às evidências obtidas por meio da análise paralela. As cargas fatoriais dos itens variaram entre 0,26 e 0,74 para os fatores considerados como representativos. A confiabilidade apurada por meio do Alfa de

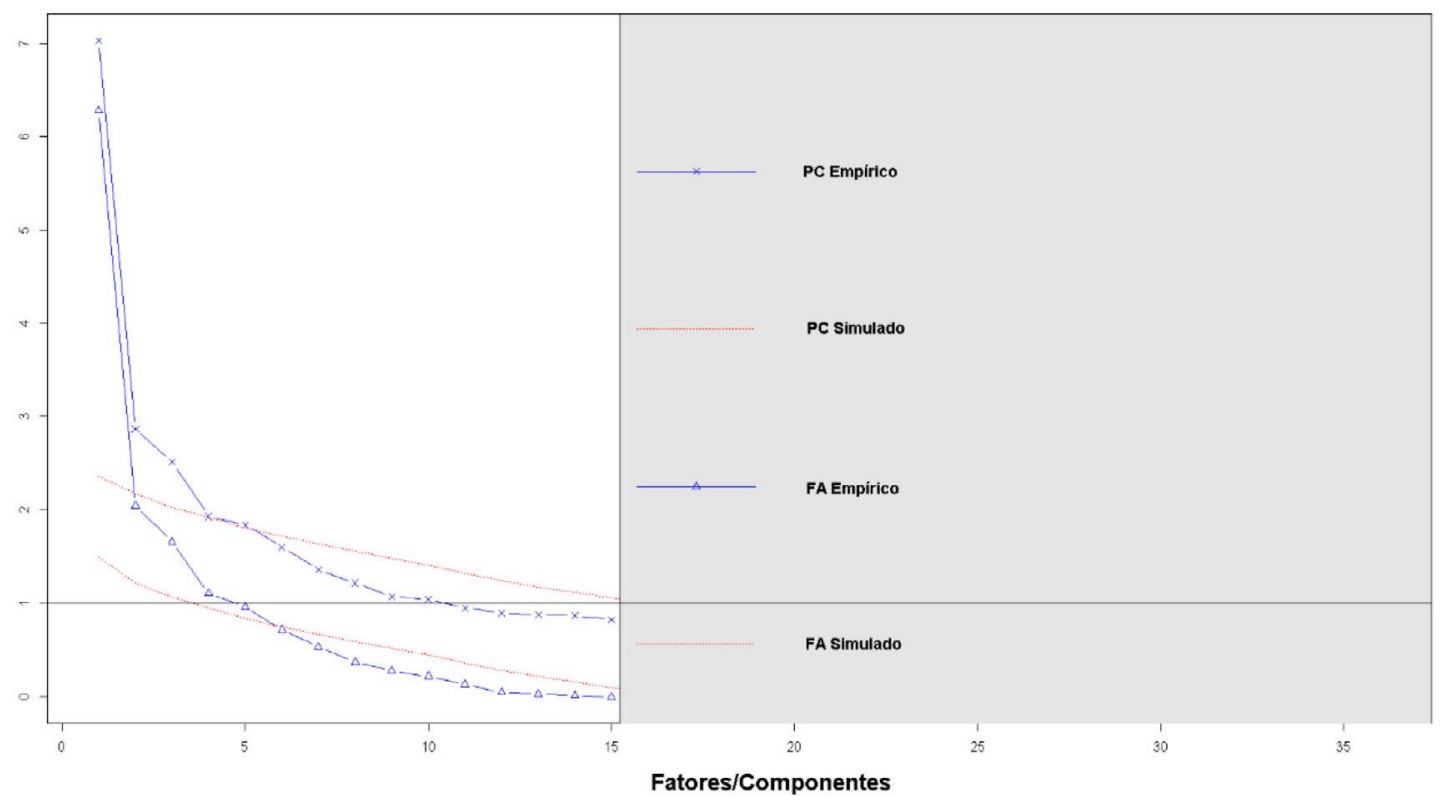

Figura 1. Representação gráfica da análise paralela contrastando autovalores empíricos e simulados 
Cronbach ajustado para dados ordinais oscilou entre 0,56 e 0,74 . No índice Omega para dados ordinais os valores variaram entre 0,62 e 0,74 . No geral, esses parâmetros foram apreendidos como razoáveis para os cinco fatores explorados na ATEBr. A solução fatorial explorada para a ATEBr é disposta na Tabela 1.

Tabela 1

Estrutura fatorial e indices de consistência interna para a ATEBr

\begin{tabular}{|c|c|c|c|c|c|}
\hline Itens - Fatores & F1 & F2 & F3 & F4 & F5 \\
\hline Item 01 - Criatividade & 0,37 & $-0,06$ & 0,20 & 0,04 & 0,08 \\
\hline Item 02 - Criatividade & 0,38 & 0,10 & $-0,22$ & 0,01 & 0,25 \\
\hline Item 03 - Criatividade & 0,50 & 0,06 & $-0,10$ & $-0,01$ & 0,12 \\
\hline Item 04 - Criatividade & 0,33 & $-0,01$ & 0,31 & $-0,16$ & 0,13 \\
\hline Item 05 - Criatividade & $-0,41$ & $-0,08$ & 0,20 & $-0,17$ & 0,18 \\
\hline Item 06 - Criatividade & $-0,34$ & 0,23 & 0,04 & $-0,03$ & 0,08 \\
\hline Item 14 - Criatividade & 0,30 & $-0,16$ & 0,15 & 0,11 & 0,14 \\
\hline Item 07 - Liderança & $-0,14$ & 0,64 & $-0,07$ & 0,04 & 0,10 \\
\hline Item 08 - Liderança & 0,01 & $-0,67$ & $-0,09$ & $-0,04$ & $-0,01$ \\
\hline Item 09 - Liderança & $-0,09$ & 0,74 & 0,03 & $-0,08$ & 0,10 \\
\hline Item 10 - Liderança & 0,17 & $-0,29$ & $-0,20$ & 0,10 & 0,01 \\
\hline Item 11 - Liderança & $-0,39$ & 0,33 & 0,28 & 0,04 & 0,05 \\
\hline Item 12 - Liderança & 0,02 & 0,41 & 0,08 & 0,02 & 0,28 \\
\hline Item 17 - Liderança & 0,00 & $-0,50$ & $-0,09$ & 0,32 & 0,15 \\
\hline Item 13 - Realização & 0,06 & 0,12 & 0,40 & 0,06 & $-0,08$ \\
\hline Item 18 - Realização & 0,09 & 0,34 & 0,38 & 0,04 & 0,05 \\
\hline Item 19 - Realização & $-0,06$ & 0,39 & 0,48 & 0,00 & $-0,01$ \\
\hline Item 20 - Realização & $-0,19$ & 0,02 & 0,48 & 0,00 & 0,42 \\
\hline Item 22 - Realização & 0,03 & 0,03 & 0,66 & $-0,05$ & $-0,14$ \\
\hline Item 23 - Realização & 0,10 & 0,30 & 0,41 & 0,01 & $-0,05$ \\
\hline Item 24 - Realização & 0,16 & 0,06 & 0,64 & 0,07 & $-0,10$ \\
\hline Item 15 - Controle & $-0,07$ & $-0,02$ & $-0,02$ & 0,69 & 0,00 \\
\hline Item 16 - Controle & $-0,02$ & 0,11 & $-0,02$ & 0,74 & $-0,06$ \\
\hline Item 26 - Controle & 0,09 & $-0,05$ & 0,37 & 0,51 & 0,05 \\
\hline Item 27 - Controle & 0,22 & 0,25 & 0,07 & 0,26 & $-0,01$ \\
\hline Item 28 - Controle & $-0,04$ & 0,09 & 0,24 & 0,26 & 0,07 \\
\hline Item 31 - Riscos & $-0,01$ & 0,27 & 0,11 & 0,17 & 0,21 \\
\hline Item 32 - Riscos & 0,05 & 0,06 & 0,20 & 0,21 & 0,31 \\
\hline Item 33 - Riscos & 0,02 & 0,04 & $-0,08$ & $-0,17$ & 0,69 \\
\hline Item 34 - Riscos & 0,08 & 0,01 & 0,03 & 0,05 & 0,71 \\
\hline Item 35 - Riscos & 0,10 & 0,03 & 0,04 & 0,03 & 0,79 \\
\hline Item 36 - Riscos & 0,22 & 0,28 & $-0,12$ & 0,07 & $\mathbf{0 , 3 0}$ \\
\hline Alfa de Cronbach (ordinal) & 0,68 & 0,56 & 0,68 & 0,71 & 0,74 \\
\hline [IC 95\%] & {$[0,63-0,74]$} & {$[0,48-0,63]$} & {$[0,62-0,73]$} & {$[0,66-0,77]$} & {$[0,70-0,79]$} \\
\hline Õmega (ordinal) & 0,68 & 0,62 & 0,62 & 0,71 & 0,74 \\
\hline [IC 95\%] & {$[0,63-0,74]$} & {$[0,56-0,69]$} & {$[0,68-0,78]$} & {$[0,66-0,77]$} & {$[0,70-0,79]$} \\
\hline
\end{tabular}

Nota. Destaques em negrito indica o fator para o qual o item foi considerado representativo. 
Cabe destacar que, após a aplicação da análise fatorial, os itens $13,14,15,16,17$ e 18 propostos para a dimensão intuição foram integrados nos demais fatores da escala por apresentarem elevada carga fatorial nesses fatores. Além disso, quatro itens propostos no modelo original da ATE não foram mantidos na estrutura fatorial da ATEBr. Os itens 25, 29 e 30 da dimensão controle apresentaram maior carregamento nos fatores criatividade e riscos, o que levou à exclusão deles. $\mathrm{O}$ item $21 \mathrm{da}$ dimensão realização também foi removido por ter carregado com maior magnitude nos fatores criatividade, riscos e liderança. Após integrar os itens da dimensão intuição aos demais fatores identificados na $\mathrm{ATEBr}$ e excluir os quatro itens que carregaram com alta magnitude em fatores distintos, a estrutura fatorial supracitada obteve os seguintes índices de ajuste para o modelo explorado $\chi 2(460)=737,10 ; p<0,01 ;$ RMSEA $=0,047$; $\mathrm{CFI}=0,900 ; \mathrm{TLI}=0,865$.

Em relação ao sentido atribuído aos fatores, o primeiro versou a respeito de elementos imaginativos, ideias e novas formas de fazer as atividades em sala, tendo seu nome fixado como criatividade. $\mathrm{O}$ segundo fator foi nomeado liderança, abrangendo itens da atitude do indivíduo sobre trabalhar em equipes e projetos na condição de líder. $O$ terceiro fator tratou sobre a atitude do sujeito frente ao esforço contínuo para alcançar o êxito e preocupação com a qualidade do trabalho, sendo identificado como realização.O quarto fator foi representado por conteúdos voltados ao quanto o indivíduo acreditava que seria bem-sucedido no futuro e poderia decidir sobre a própria carreira, sendo denominadocontrole.O quinto fator centrou-se em conteúdos atitudinais a respeito de investimentos em situações inovadoras e incertas, o qual foi identificado como riscos. A estrutura fatorial encontrada teve suas particularidades discutidas adiante.

\section{Discussão}

O presente trabalho teve como objetivo apresentar evidências de validade empírica baseada na estrutura interna para a ATEBr (Attitude Towards Entrepreneurship -Brazil). Comparando-se a estrutura fatorial da versão adaptada da escala (ATEBr) com a versão original (ATE) nota-se que a adaptação brasileira replicou a maior parte da estrutura identificada em língua inglesa (Athayde, 2009), consolidando na literatura brasileira um modelo capaz de replicar a avaliação da atitude empreendedora pelo modelo tripartite de atitudes de forma multifatorial. Os fatores criatividade, liderança e realização foram confirmados integralmente, ao passo que modificações pontuais aconteceram no fator controle. Também foi identificado o fator riscos na estrutura fatorial da ATEBr, o qual compõe as dimensões teorizadas por Athayde (2009) para atitude empreendedora, mas não foi encontrado de forma empírica no estudo original da autora com estudantes ingleses.
Sobre as modificações realizadas no fator controle, salienta-se que esse fator teve itens excluídos por particularidades semânticas e culturais relativas ao processo de adaptação da escala que descaracterizaram os itens como controle sobre a carreira. No processo de adaptação da escala, os itens 25,29 e 30 que contavam com vocábulos "job" e "work", foram traduzidos para "emprego" e "profissional". Essa particularidade na tradução fez com que esses itens se diferenciassem do conteúdo expresso nos itens 26,27 e 28 retidos na estrutura fatorial, os quais tiveram o vocábulo "career" adaptado para "carreira".

Considerando-se que na ATEBr o fator controle refere-se primordialmente à carreira como futuro empreendedor de forma mais expandida, conteúdos relativos ao controle de aspectos profissionais ou emprego descaracterizam o fator em avaliação por esses vocábulos se apresentarem mais específicos, o que explica a diferença semântica supracitada. No Brasil, o termo "profissional" pode remeter ao exercício da atividade técnica para o qual o estudante recebe treinamento no curso superior, sendo, portanto, conteúdo distinto do empreendedorismo. Por sua vez, a palavra "emprego" tende a se associar com vínculos de trabalhos em que o indivíduo se insere em uma organização desenvolvida por outro agente empreendedor como colaborador. Nesse sentido, considerou-se adequada a exclusão desses itens pelo aspecto semântico ter distorcido a proposta de avaliação.

Adicionalmente, cabe destacar que a integração dos itens relativos à dimensão intuição nos fatores criatividade, liderança, realização e controle parece indicar que a intuição não se propõe como fator isolado no modelo teórico da atitude empreendedora. Nesse sentido, utilizar da intuição no contexto do empreendedorismo apresenta-se como uma atitude transversal que perpassa os demais fatores. No fator criatividade, a intuição representou conteúdos favoráveis a cometer erros como forma de resolver problemas de maneiras inusitadas por meio do item 14. No fator liderança o conteúdo do item 17 advindo da dimensão intuição apresentou carga negativa, indicando que a atitude frente à liderança tende a ser mais sistematizada do que intuitiva no contexto do empreendedorismo, não permitindo ao empreendedor intuir sobre o que não sabe quando está agindo de forma persuasiva frente aos demais.

Ainda sobre a adição do conteúdo relativo à intuição em outros fatores, a contribuição do conteúdo do item 13 para o fator realização demonstrou que parte da atitude do empreendedor em busca do êxito se refere ao fato de o indivíduo intuir que é possível encontrar respostas e soluções sem conhecer completamente os fatos relacionados à solução de um problema. Por sua vez, os itens 15 e 16 da dimensão intuição acrescentaram ao fator controle conteúdos relativos à confiança de que o julgamento pessoal e os instintos são úteis para resolver problemas e, portanto, 
permitir ao indivíduo maior controle sobre seu futuro e carreira.

Cabe destacar que outros dois estudos realizados com estudantes da África do Sul também não confirmaram o fator intuição como dimensão isolada (Maluleke, 2011; Steenekamp, van der Merwe, \& Athayde, 2011), o que reforça a necessidade de reexaminar a pertinência teórica e empírica dessa dimensão. No presente estudo, a opção de manter os itens com conteúdo de intuição nos demais fatores respaldou-se em compreensões teóricas de que a intuição é relevante no processo de empreender, mas ainda há dificuldades em operacionalizá-la de forma isolada (Baldacchino, Ucbasaran, Cabantous, \& Lockett, 2015; Michell, Friga, \& Mitchell, 2005). A maior diferença encontrada entre a proposta original e a versão adaptada da medida refere-se à confirmação do fator riscos na $\mathrm{ATEBr}$, o qual é proposto como dimensão teórica no estudo inglês, mas não foi suportado pelas evidências empíricas geradas na Inglaterra para a ATE (Athayde, 2009).

Sobre esse aspecto hipotetiza-se que a identificação do fator riscos na ATEBr reside nas diferenças culturais entre Inglaterra e Brasil no que tange ao nível de desenvolvimento social e econômico. Em países emergentes, tal como o Brasil, há uma relação positiva entre o nível de risco assumido pelos indivíduos e o exercício empreendedor pela ausência de políticas públicas que ofereçam suporte à proposição do próprio negócio (Freytag \& Thurik, 2007). Nesse cenário, parece relevante para o futuro empreendedor brasileiro, inserido numa economia emergente, a disposição para assumir riscos, o que justifica a inclusão desse fator na ATEBr. Afinal, na ausência de políticas e incentivos públicos sobre o tema, o brasileiro precisa enfrentar mais riscos para empreender quando comparado com indivíduos situados na Inglaterra (Grilo \& Irigoyen, 2006). Isso acontece pois o delineamento de políticas públicas que oferecem suporte à proposição do próprio negócio em países como a Inglaterra dilui o risco associado ao empreendimento (Mota, Sobreira, Vale, \& Nogueira, 2017).

Ademais, partindo-se das evidências geradas no presente estudo para a medida, é possível vislumbrar a aplicação da ATEBr nas práticas de orientação profissional brasileiras como um instrumento voltado à Empregabilidade e interesse de forma restrita. Isto é, após a aplicação dos instrumentos de interesses profissionais e Empregabilidade que rastreiam entre fatores ocupacionais gerais, indivíduos identificados como possíveis interessados pelo empreendedorismo podem ter o processo de orientação otimizado ao se aplicar a ATEBr. Com a ATEBr é possível mapear a atitude individual frente a aspectos que se mostram relevantes no contexto do empreendedorismo como criatividade, liderança, realização, controle e riscos. Nesse caso, se o indivíduo apresentar baixo escore em algum dos fatores da ATEBr, torna possível planejar um processo de orientação profissional que busque maximizar a atitude pessoal do sujeito frente ao fator deficitário que se propõe como relevante para caracterizar a atitude empreendedora, o que demonstra o potencial de aplicação do instrumento frente às propostas existentes na literatura.

\section{Considerações finais}

A presente investigação teve como objetivo investigar evidências de validade empírica baseada na estrutura interna para a $\mathrm{ATEBr}$, a fim oferecer contribuições para a otimizar as condições de mensuração da atitude empreendedoraentre estudantes universitários. Pelas evidências apresentadas, nota-se que aATEBr contribui à literatura nacional ao se propor como uma medida com modelo teórico robusto, cujas evidências de validade devem ser expandidas em estudos ulteriores. Em relação ao problema elencado na presente investigação, nota-se que comparativamente à IMAEa ATEBr foi capaz de sustentar uma estrutura multifatorial para avaliação da atitude empreendedora de forma específica no contexto brasileiro. Nesse sentido, ao comparar a ATEBr com as propostas de avaliação de interesse e Empregabilidade no Brasil, nota-se complementariedade entre os conceitos: a atitude empreendedora pode facilitar a identificação de atitude pessoais relativas ao interesse individual por empreender com maior ênfase nos fatores que se relacionam com o empreendedorismo.

Ao contrastar a ATEBr com as propostas de mensuração do empreendedorismo existentes na literatura nacional também se nota um avanço na perspectiva analítica, devido ao uso estimadores e técnicas de análise robustas a dados ordinais, o que deve ser empregado em estudos futuros de avaliação do fenômeno com escalas de resposta do tipo Likert. Em uma perspectiva prática, a contribuição da $\mathrm{ATEBr}$ refere-se à possibilidade de avaliação da atitude empreendedora em língua portuguesa por um modelo transcultural que pode viabilizar a comparação entre diferentes países, tendo em vista a existência de convergências na estrutura fatorial da ATEBr com versões da ATE geradas em outras localidades (Athayde, 2009; Maluleke, 2011; Spagnoli, Santos, \& Caetano, 2017).

Para desenvolvimentos futuros, sugerem-se investigações que busquem confirmar a estrutura fatorial da escala e apreender relações da medida com outras variáveis.Destaca-se como limitação da investigação a aplicação da medida somente em uma única universidade pública, o que limita as evidências geradas para esse contexto. Assim, torna-se fundamental a elaboração de estudos que proponham novas evidências para a ATEBr, principalmente em outros ambientes universitários públicos e privados, espaços de ensino técnico-profissionalizante e diferentes instituições de educação formal e não-formal voltadas para o aprimoramento das atitudes 
individuais associadas ao empreendedorismo (Ambiel, 2017; Henrique \& Cunha, 2008).

Por fim, entende-se que a compreensão de um tema complexo, tal como as características pessoais dos empreendedores, torna-se ainda mais impactante quando diferentes pares interagem na produção do conhecimento. Afinal, é por meio da interlocução entre aqueles que se propõem a compreender os conceitos relacionados ao empreendedorismo que fontes de evidências empíricas podem ser contrastadas, a fim de otimizar as possibilidades de mensuração e avaliação do fenômeno nos contextos de orientação profissional que aventem o empreendedorismo como alternativa ocupacional.

\section{Referências}

Ajzen, I., \& Fishbein, M. (2005). The Influence of Attitudes on Behavior. In D. Albarracín, B. T. Johnson, \& M. P. Zanna (Eds.), The Handbook of Attitudes (pp. 173-221). Mahwah, NJ: Erlbaum.

Ambiel, R. A. M., Campos, M. I., \& Campos, P. P. V. Z. (2017). Análise da Produção Científica Brasileira em Orientação Profissional: Um Convite a Novos Rumos. Psico-USF, 22(1), 133-145. doi:10.1590/1413-82712017220112.

Ambiel, R. A. M., Lamas, K. C. A., \& Silva, L. L. M. (2016). Avaliação dos Interesses Profissionais no Brasil: revisão da produção científica. Avaliação Psicológica, 15(1), 1-9.

Athayde, R. (2009). Measuring enterprise potential in young people. Entrepreneurship Theory and Practice, 33(2), 481-500.

Baldacchino, L., Ucbasaran, D., Cabantous, L., \& Lockett, A. (2015). Entrepreneurship research on intuition: a critical analysis and research agenda. International Journal of Management Reviews, 17(2), 212-231. doi:10.1111/ijmr.12056

Campos, K. C. D. L. (2011). Employability scale construction: definitions and psychological variables. Estudos de Psicologia (Campinas), 28(1), 45-55.

Carland III, J. W., Carland Junior, J. W., Carland, J. A. C., \& Pearce, J. W. (1995). Risk taking propensity among entrepreneurs, small business owners and managers. Journal of Business and Entrepreneurship, 7(1), 15-23.

Cesário, F. S., \& Feijão, A. M. P. (2014). Impacto das percepções de Empregabilidade num contexto de insegurança de emprego. Revista Psicologia Organizações e Trabalho, 14(1), 89-103.

Chaiken, S., \& Stangor, C. (1987). Attitudes and attitude change. Annual Review of Psychology, 38(1), 575-630.

CNS - Conselho Nacional de Saúde. (2016). Resolução $N^{o}$ 510/2016. Recuperado de http://conselho.saude.gov.br/resolucoes/2016/Reso510.pdf

Cortez, P. A., \& Veiga, H. M. S. (2018). Características pessoais dos empreendedores: clarificação conceitual dos construtos e definições da literatura recente (2010-2015). Estudos Interdisciplinares em Psicologia, 9(3), 58-79.

Cortez, P.A. (2017). Evidências de validação empírica de escala de atitude empreendedora e testagem de um modelo preditivo a partir dos Cinco Grandes fatores de personalidade (Dissertação). Recuperado de https://repositorio.ufu. br/handle/123456789/18387

Costa-Nogami, V. K., \& Machado, H. V. (2014). Análise da atividade empreendedora dos países Ibero Latinoamericanos de acordo com o Global Entrepreneurship Monitor (GEM). Latin American Journal of Business Management, 5(1), 83-108.

Damásio, B. F. (2012). Uso da análise fatorial exploratória em psicologia. Avaliação Psicológica, 11(2), 213-228.

Draghici, A., Albulescu, C. T., \& Tamasila, M. (2014). Entrepreneurial attitude as knowledge asset: its impact on the entrepreneurial activity in Europe. Procedia Social and Behavioral Sciences, 109(1), 205-209. doi:10.1016/j. sbspro.2013.12.445

Epstein, S. (1984). A procedural note on the measurement of broad dispositions. Journal of Personality, 52(4), 318-325.

Frese, M., \& Gielnik, M. M. (2014). The psychology of entrepreneurship. Annual Review of Organizational Psychology and Organizational Behavior, 1(1), 413-438. doi:0.1146/annurev-orgpsych-031413-091326 
Freytag, A., \& Thurik, R. (2007). Entrepreneurship and its determinants in a cross-country setting. Journal of Evolutionary Economics, 17(2), 117-131.

Gibb, A. (1987). Enterprise culture-its meaning \& implications for education and training. Journal of European Industrial Training, 11(3), 2-38.

Goulart, C. S., \& Kruger, C. (2016). Atitude empreendedora: uma análise da produção científica na área de administração na Web of Science no período de 2005 a 2014. Revista Estudo \& Debate, 23(1), 122-137.

Grilo, I., \& Irigoyen, J. M. (2006). Entrepreneurship in the EU: to wish and not to be. Small Business Economics, 26(4), 305-318.

Henrique, D. C., \& Cunha, S. K. (2008). Práticas didático-pedagógicas no ensino de empreendedorismo em cursos. RAM - Revista de Administração Mackenzie, 9(5), 112-136.

Inácio, E., Machado, H. V., Gimenez, F. A., \& Morini, C. (2014). From 1980 to 2010: An Overview about the Brazilian Scientific Production in Entrepreneurship. International Journal of Entrepreneurship, 18, 129-141.

Ismail, A. (2015). The Entrepreneurial Attitude and Intentions of Newly Enrolled University Students-Issues and Policy Implications. Journal of Research in Business, Economics and Management, 4(3), 426-436.

Johnson, J., \& Mathew, S. K. (2017). Entrepreneurial Attitudes and Their Influence on the Entrepreneurial Intention. Asian Journal of Research in Business Economics and Management, 7(8), 261-274.

Krueger, N. F., Reilly, M. D., \& Carsrud, A. L. (2000). Competing models of entrepreneurial intentions. Journal of Business Venturing, 15(5), 411-432.

Krueger, N. F. \& Brazeal, D.V. (1994). Enterprise potential and potential entrepreneurs. Entrepreneurship Theory and Practice, 18(3), 91-104.

Lindzey, G., Gilbert, D., \& Fiske, S. T. (1998). The Handbook of Social Psychology. New York, NY, Oxford University Press.

Llewellyn, D. J., \& Wilson, K. M. (2003). The controversial role of personality traits in entrepreneurial psychology. Education+ Training, 45(6), 341-345.

Lopes Júnior, G. S., \& Souza, E. C. L. (2005). Atitude empreendedora em proprietários-gerentes de pequenas empresas. Construção de um instrumento de medida. Revista Eletrônica de Administração, 11(6), 1-21.

Louw, L., Van Eeden, S. M., Bosch, J. K., \& Venter, D.J.L. (2003). Entrepreneurial traits of undergraduate students at selected South African tertiary institutions. International Journal of Entrepreneurial Behaviour \& Research, 9(1), $5-26$.

Maluleke, R. (2011). Entrepreneurial attitude of rural secondary school learners in an emerging economy. Tese de Doutorado. Gordon Institute of Business Science, University of Pretoria, África do Sul.

Manning, L., \& Parrott, P. (2017). The impact of workplace placement on students' entrepreneurial attitude. Higher Education, Skills and Work-Based Learning, 8(1), 56-69.

Mitchell, J. R., Friga, P. N., \& Mitchell, R. K. (2005). Untangling the intuition mess: Intuition as a construct in entrepreneurship research. Entrepreneurship Theory and Practice, 29(6), 653-679.

Mota, M. O., Sobreira, M., C., Vale, M. S., \& Nogueira, L. C. C. (2017). Relações de influência de indicadores macroeconômicos na propensão ao risco de empreender. REGE-Revista de Gestão, 24(2), 159-169.

Oliveira, B. M. D. F., Vieira, D. A., Laguía, A., Moriano, J. A., \& Salazar-Soares, V. J. (2016). Intenção empreendedora em estudantes universitários: adaptação e validação de uma escala (QIE). Avaliação Psicológica, 15(2), 187-196.

Pasquali, L. (2015). Delineamento de Pesquisa em Ciência: Fundamentos Estatísticos da Pesquisa Científica (vol. 2). São Paulo: Vetor.

Peixoto, A. L. A., Souza, J. A. J., Aguiar. A. C. V. N. Autopercepção de Empregabilidade . In: K.P. Palácios \& A. L. A. Peixoto (Orgs.), Ferramentas de Diagnóstico para Organizações e Trabalho: Um Olhar a Partir da Psicologia (1a ed, Vol. 1, pp.175-186). Porto Alegre: Artmed. 
Rauch, A., \& Frese, M. (2000). Psychological approaches to entrepreneurial success: A general model and an overview of findings. International Review of Industrial and Organizational Psychology, 15(1), 101-142.

Robinson, J. P., Shaver, P. R., \& Wrightsman, L. S. (2013). Measures of Personality and Social Psychological Attitudes: Measures of Social Psychological Attitudes. Academic Press.

Robinson, P. B., Stimpson, D. V., Huefner, J. C., \& Hunt, H. K. (1991). An attitude approach to the prediction of entrepreneurship. Entrepreneurship Theory and Practice, 15(4), 13-31.

Rounds, J., \& Su, R. (2014). The nature and power of interests. Current Directions in Psychological Science, 23(2) 98-103.

Schmidt, S., \& Bohnenberger, M. C. (2009). Perfil empreendedor e desempenho organizacional. Revista de Administração Contemporânea, 13(3), 450-467.

Souza, D. L., Souza, J. B., Pasin, L. E. V., \& Zambalde, A. L. (2016). Empreendedorismo e desenvolvimento local: uma análise do programa Microempreendedor Individual em Minas Gerais, Brasil. Desenvolvimento em Questão, 14(37), 262-292.

Souza, E. C. L., Lopez Júnior, G. S., Bornia, A. C., \& Alves, L. R. R. (2013). Atitude empreendedora: validação de um instrumento de medida com base no modelo de resposta gradual da teoria da resposta ao item. RAM Revista de Administração Mackenzie, 14(5), 230-251.

Souza, G. H. S. D., Santos, P. D. C. F. D., Lima, N. C., Cruz, N. J. T. D., Lezana, Á. G. R., \& Coelho, J. A. P. D. M. (2017). Entrepreneurial Potential Scale: evidence on confirmatory factor validity, dimensional structure and predictive effectiveness. Gestão \& Produção, 24(2), 324-337.

Spagnoli, P., Santos, S., \& Caetano, A. (2017). Adaptation of the attitude toward enterprise test in the italian high school and university contexts. Journal of Developmental Entrepreneurship, 22(1), 1-16. doi:10.1142/S1084946717500054

Steenekamp, A. G., van der Merwe, S. P., \& Athayde, R. (2011). Application of the attitude toward enterprise (ATE) test on secondary school learners in south africa. SAJEMS NS, 14(2), 314-332.

Strauss, A. (1988). The articulation of project work: An organizational process. The Sociological Quarterly, 29(2), 163-178.

Vecchio, R.P. (2003). Entrepreneurship and leadership: Some common trends and threads. Human Resource Management Review, 13(1), 303-327.

Recebido: $13 / 07 / 17$

Aceito: $28 / 11 / 19$

Sobre os autores:

Pedro Afonso Cortez é Doutorando em Psicologia pela Universidade São Francisco. Mestre em Psicologia pela Universidade Federal de Uberlândia. Especialista em Psicologia Organizacional e do Trabalho pela Faculdade de Administração, Ciências, Educação e Letras. Psicólogo com ênfase em Psicologia Clínica, Social e Processos de Gestão pela Universidade Federal de Uberlândia.

Heila Magali da Silva Veiga é Docente vinculada ao Instituto de Psicologia da Universidade Federal de Uberlândia. Doutora em Psicologia Social, do Trabalho e das Organizações pela Universidade de Brasília. Mestre em Psicologia e Psicóloga pela Universidade de Brasília. Graduada em Administração Pública pela Universidade Federal de Uberlândia.

Jonilto Costa Sousa é Docente vinculado ao Instituto de Educação Superior da Universidade de Brasília. Doutor em Administração pela Universidade de Brasília. Mestre em Administração pela Universidade de Brasília. Especialista em Inteligência Organizacional e Competitiva pela Universidade de Brasília. Graduado em Administração pelo Centro Universitário do Distrito Federal. 
